\title{
Leishmania (Viannia) braziliensis transfectants overexpressing the miniexon gene lose virulence in vivo
}

\author{
Juliano Simões de Toledo a , André F. Junqueira dos Santos a, Tatiana Rodrigues de Moura ${ }^{\text {b }}$ \\ Simone Aparecida Antoniazi ${ }^{\text {, }}$, Cláudia Brodskyn ${ }^{\text {b,c }}$, Camila Indiani de Oliveira ${ }^{\text {b }}$, \\ Aldina Barral $^{\text {b,c }}$, Angela Kaysel Cruz ${ }^{\text {a,* }}$ \\ ${ }^{a}$ Departamento de Biologia Celular e Molecular e Bioagentes Patogênicos, Faculdade Medicina de Ribeirão Preto-Universidade de São Paulo, Ribeirão Preto, São Paulo, Brazil \\ b Centro de Pesquisas Gonçalo Moniz, FIOCRUZ, Salvador, Bahia, Brazil \\ c Instituto de Investigação em Imunologia, Salvador, Bahia, Brazil
}

\section{A R T I C L E I N F O}

\section{Article history:}

Received 1 August 2008

Received in revised form 25 September 2008

Accepted 26 September 2008

Available online 25 October 2008

\section{Keywords:}

Leishmania (V.) braziliensis

Virulence

Miniexon

Overexpression

SL RNA

\begin{abstract}
A B S T R A C T
The miniexon gene has a central role in the processing of polycistronic pre-mRNA of kinetoplastids. It is added to the 5' extremity of each mRNA, supplying the 5'-capped structure to the molecule. Previous studies in Leishmania (Leishmania) major showed that the overexpression of the miniexon array attenuates the virulence of the parasite in in vivo assays. The results presented here extend those findings to Viannia subgenus. Leishmania (Viannia) braziliensis was transfected with a cosmid harboring a tandem array of one hundred miniexon gene copies and then characterized by Northern blot analysis. The overexpression of the exogenous gene was confirmed and its effect on the virulence of $L .(V$.) braziliensis was investigated in hamsters. In BALB/c mice we could not detect parasites during the course of 15 weeks of infection. In addition, hamsters infected with transfectants overexpressing the miniexon gene exhibited only a minor footpad swelling of late onset and failed to develop progressive lesion, these attenuated parasites could be recovered from the inoculation site 1 year after infection. The persistence of parasites in the host indicates that a stable line overexpressing the miniexon may be tested as live vaccine against leishmaniasis.
\end{abstract}

(c) 2008 Elsevier Ireland Ltd. All rights reserved.

\section{Introduction}

Leishmaniasis is a spectrum of diseases (cutaneous, mucocutaneous, and visceral) caused by parasitic protozoan of the genus Leishmania. This parasite goes through two basic forms: the flagellated promastigote, which proliferates in the gut of a sand fly and is transmitted to the mammalian host by the bite of the insect vector, and the amastigote form, which replicates and survives within the phagolysosome of the host macrophage [1]. The variety of biological novelties detected in this ancient eukaryote and its medical relevance have led several research groups to investigate the biology and molecular processes it undergoes. The parasite has unusual mechanisms for the flow and control of gene expression, such as polycistronic transcription, trans-splicing and RNA editing [reviewed by 2,3]. Messenger RNA synthesis in kinetoplastids

Abbreviations: SL RNA, Spliced leader RNA; bp, Base pairs; nt, Nucleotides; kb, Kilobase pairs; HEPES, N-2 hydroxyethylpiperazine-N\%-2-ethanesulfonic acid; ME, Miniexon; LNs, Lymph nodes.

* Corresponding author. Departamento de Biologia Celular e Molecular e Bioagentes Patogênicos, Faculdade Medicina de Ribeirão Preto, Universidade de São Paulo, Av. Bandeirantes, no. 3900, CEP 14049-900 Ribeirão Preto, SP, Brazil. Tel.: +55163602 3318; fax: +551636336631.

E-mail address: akcruz@fmrp.usp.br (A.K. Cruz). protozoa involves the addition of a spliced leader (a 39-nucleotide capped miniexon) sequence to most, if not all, maturing mRNA. The spliced leader is initially transcribed as a 140-nucleotide miniexonderived RNA [4] and the transfer of the 5'-capped 39-nucleotide miniexon to the $5^{\prime}$-end of each pre-mRNA occurs by a bimolecular transsplicing reaction.

The miniexon gene (ME) is organized in a tandem array in the genome of all trypanosomatids and its sequence and extension varies among species [4-6]. Nevertheless, its overall structure is conserved, containing two functional domains: the exon, highly conserved, and the intron, which needs a conserved secondary structure to be functional. These functional domains are followed by a variable untranscribed region. In Leishmania major the array resides on chromosome 2 and the number of repeats, which are $455 \mathrm{bp}$ long, varies among different strains [4-9].

The miniexon gene has a central role in the RNA processing reaction of kinetoplastids protozoa. It is required for RNA maturation, transcript stability and initiation of translation. The parasite is unable to survive in the absence of miniexon transcription [10-12]. Iovannisci and Beverley associated expansion of the miniexon array with growth advantage of promastigotes in vitro [13]. Samaras and Spithill showed that an avirulent $L$. (L.) major clone presented a larger chromosome 2 and suggested that its size polymorphism could be due to an expansion of the miniexon locus [11]. On the other hand, a few L. (L.) major 


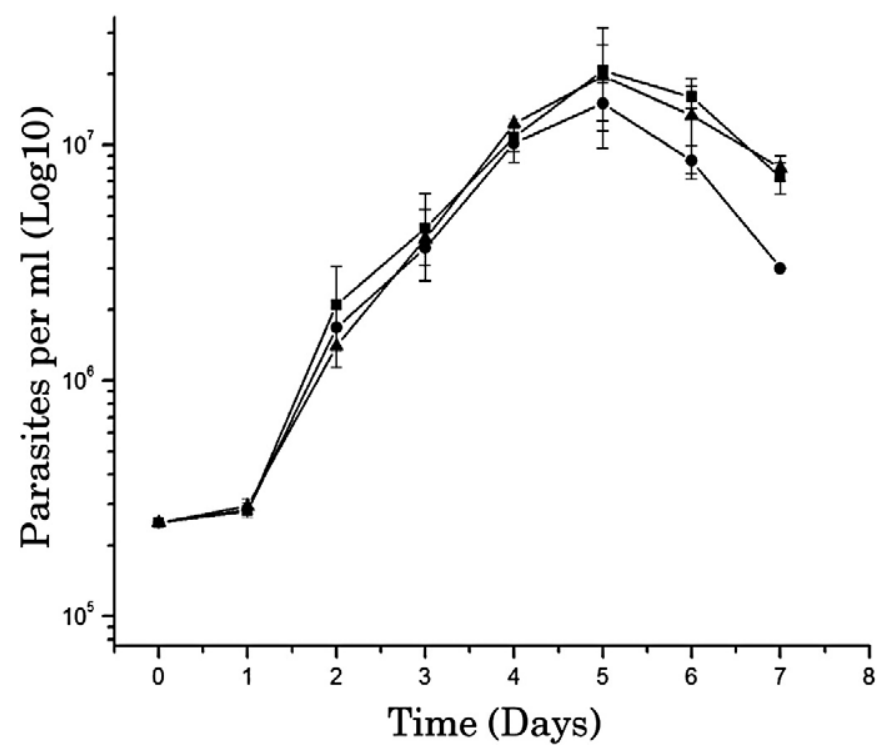

Fig. 1. Growth curve of $L$. (V.) braziliensis parental and transfectant lines. Parental line was grown in M199 supplied with $2 \%$ of human urine and the transfectants were cultured with $120 \mu \mathrm{g} / \mathrm{ml}$ of hygromycin B. The number of parasites was estimated using a hemocytometer. L. (V.) braziliensis parental line (square); Lb [CLHYG $\left.{ }^{120}\right]$ (triangle); Lb $\left[{ }^{2} L H Y G^{120} M E\right]$ (circle).

virulent lines, such as Friedlin and LV39, bear an amplified version of one or both alleles of chromosome 2 [14]. Antoniazi et al. demonstrated that virulent $L$. (L.) major lines lose virulence in in vivo infection assays after transfection of a recombinant cosmid bearing only the miniexon gene array. Overexpressors "cured" of the circular molecule reverted to the virulent phenotype, which confirms a positive correlation between overexpression of the miniexon and loss of virulence [15].

In this study we show that hamsters infected with a Leishmania (V.) braziliensis strain overexpressing the $L$. (L.) major miniexon array ( $c L H Y G M E$ ) does not develop progressive lesions. The remarkable phenomenon of virulence attenuation observed in $L$. $(V$.) braziliensis indicates that overexpression of the miniexon gene affects the outcome of infection.

\section{Materials and methods}

\subsection{Cell lines, culture conditions and transfection}

Clonal virulent L. (V.) braziliensis H3227 (MHOM/BR/94/H-3227) was used to host the miniexon recombinant as an extrachromosomal molecule. Cells were grown in M199 medium supplemented with 10\% fetal bovine serum, $2 \%$ human urine, $100 \mathrm{mM}$ adenine, $10 \mathrm{mg} / \mathrm{ml}$ hemin, $40 \mathrm{mM}$ HEPES (pH 7.4), 50 units/ml penicillin and $50 \mathrm{mg} / \mathrm{ml}$ streptomycin. Cells grown to late log phase were transfected by electroporation $(500 \mu \mathrm{F}$ and voltage $2.25 \mathrm{kV} / \mathrm{cm})$ and stable transfectants were selected in semi-solid medium ( $32 \mu \mathrm{g} / \mathrm{ml}$ hygromycin B), as described previously by Kapler et al. [16]. Transfectants were named according to Clayton et al. [17] and drug concentration used in liquid culture $(\mu \mathrm{g} / \mathrm{ml})$ is indicated by superscript. The control line carrying the cosmid with no insert and ME overexpressor bearing one hundred copies of the $L$. (L.) major ME array were named $\mathrm{Lb}$ [CLHYG] and $\mathrm{Lb}$ [CLHYG ME] respectively. We used the parasites cultured in liquid medium at drug concentration of 12 and $120 \mu \mathrm{g} / \mathrm{ml}$ hygromycin B in order to compare the effect of heterologous ME overexpression under two different drug pressure conditions.

\subsection{Total RNA preparation}

Total RNA was prepared with Trizol reagent (Invitrogen). Samples were fractionated by either denaturing $1 \%$ agarose gel or denaturing $12 \%$ polyacrylamide gel electrophoresis, blotted to nylon membranes (GeneScreen Plus, Dupont) and hybridized following standard methods [18].

\subsection{DNA labeling}

A PCR-amplified fragment of the hygromycin phosphotransferase gene ( $h y g$ ) and a 455 bp ME fragment derived from a Not I digestion of 32D05 (the cosmid bearing the miniexon array) [15], were used as templates for a random priming labeling reaction $[19,20]$ with $\left[\alpha-{ }^{32} \mathrm{P}\right]$ dCTP (GE Healthcare, Piscataway, PA, USA) to produce probes for Northern experiments.
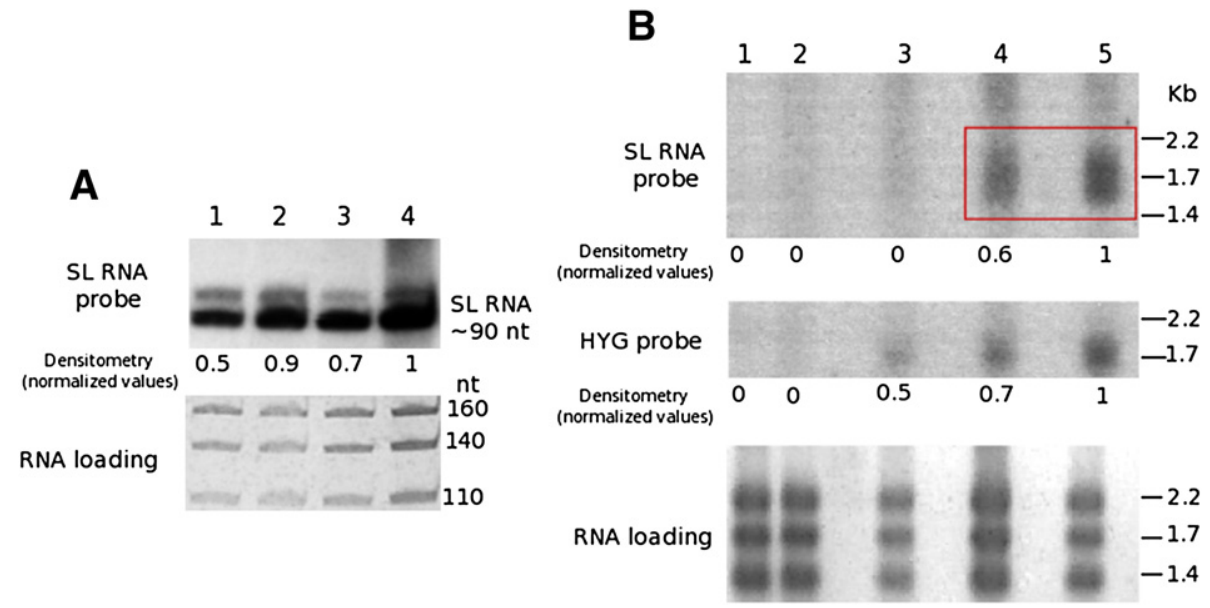

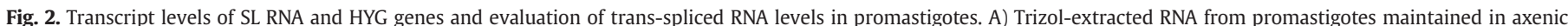

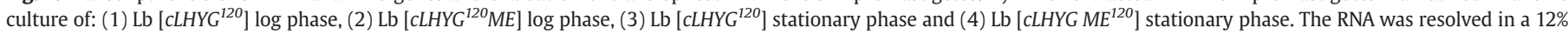

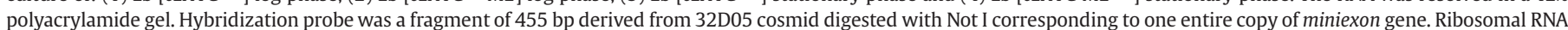

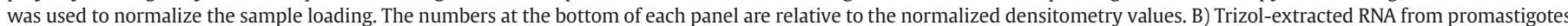

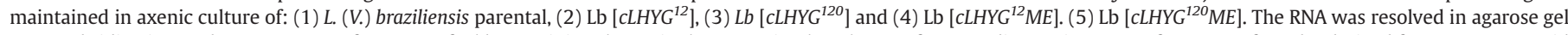

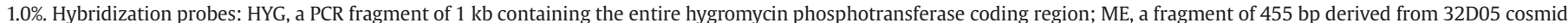

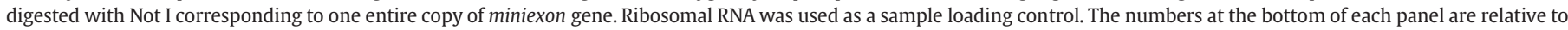

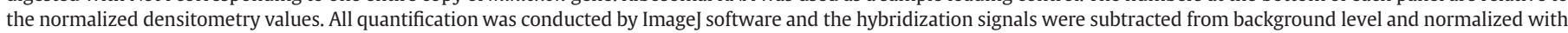
sample loading. 
A

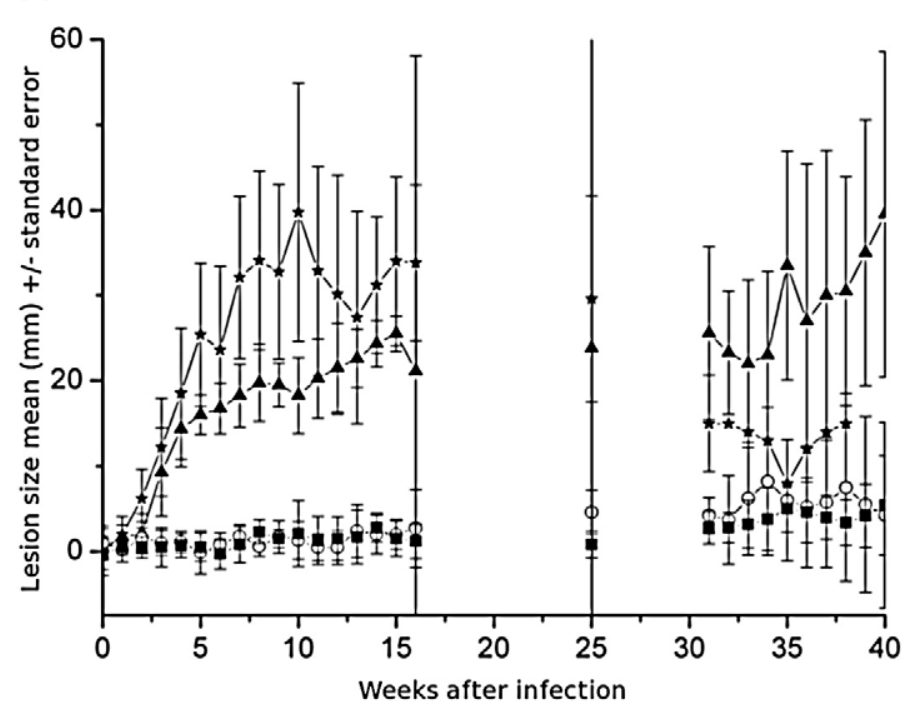

B
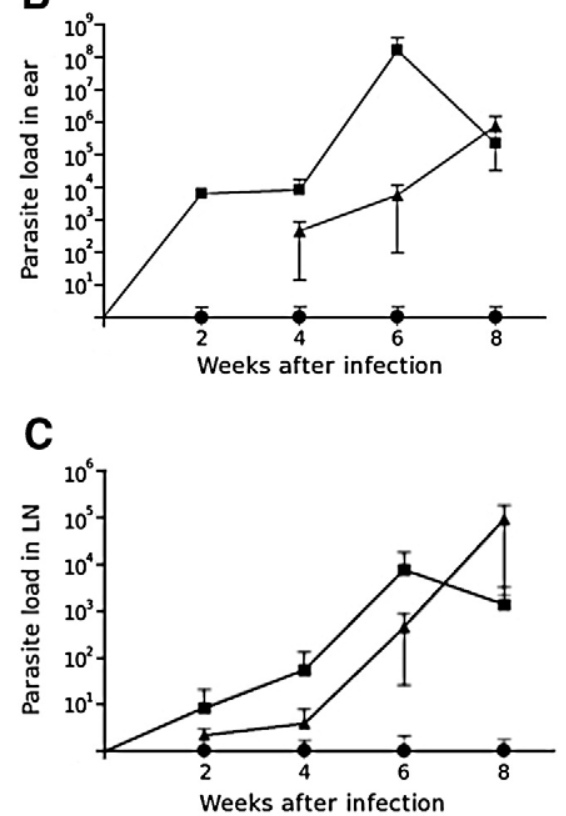

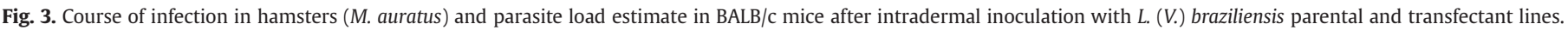

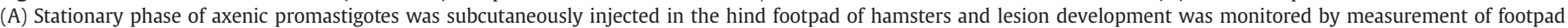

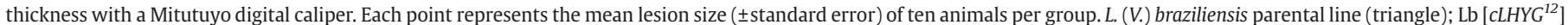

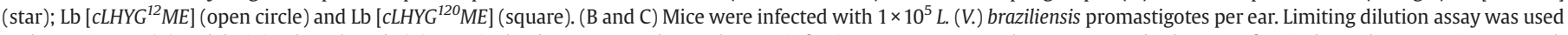

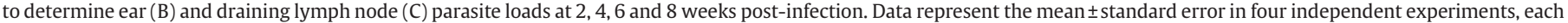
performed on six mice. L. (V.) braziliensis parental line (square), Lb $\left[c L H Y G^{120}\right]$ (triangle), Lb $\left[c L H Y G^{120} M E\right]$ (circle).

\subsection{Animals, parasite inoculation and lesion measurement}

Hamsters were obtained from an animal facility in the Medical School (FMRP-USP, Ribeirão Preto, SP, Brazil). Stationary-phase $L$. (V.) braziliensis $\left(10^{7}\right.$ viable parasites in $25 \mu$ l of saline) promastigotes were inoculated subcutaneously into the right hind footpads of hamsters ( $n=10$ per experiment). Lesion progression was recorded once a week by measuring footpad swelling (dorsal to plantar axis) with a vernier caliper, using the non-infected contra-lateral rear footpad as a control. One year after infection, footpads from five hamsters were harvested for RNA extraction and Northern analysis. Female BALB/c mice (4-8 weeks old) were obtained from the Multidisciplinary Center for Biological Investigation (CEMIB-UNICAMP, Campinas, SP, Brazil). Stationary-phase promastigotes $\left(10^{5}\right.$ viable parasites in $10 \mu \mathrm{l}$ of saline) were inoculated into the right ear dermis of age-matched BALB/c mice using a 27.5-gauge needle ( $n=5$ per experiment) [25]. Lesion size was monitored weekly for 10 weeks with a digital caliper (Thomas Scientific, Swedesboro, NJ).

\subsection{Parasite load estimate}

Infected ears and retromaxillar draining lymph nodes (LNs) were aseptically excised from BALB/c mice at 2, 4, 6 and 8 weeks postinfection and homogenized in Schneider's medium (Sigma Chemical Co., St. Louis, MO). The homogenates were serially diluted in Schneider's medium supplemented with $10 \%$ fetal bovine serum and $2 \%$ human urine, and distributed into 96-well plates containing biphasic blood agar (Novy-Nicolle-McNeal) medium. Parasite load was determined using a quantitative limiting dilution assay as described previously [21].

\subsection{Culture and infection of J774 macrophages}

Cultures of $\mathrm{J774}$ macrophages were maintained in RPMI medium supplemented with $10 \%$ of bovine fetal serum at $37{ }^{\circ} \mathrm{C}$ in $5 \% \mathrm{CO}_{2} . \mathrm{J} 774$ cells were infected with $L$. $(V$. $)$ braziliensis in a ratio of $1: 5$ for $3 \mathrm{~h}$ at room temperature.

\subsection{Electron microscopy}

For transmission electron microscopy, $2 \times 10^{6}$ J774 macrophages were pelleted at $1000 \times g$, washed twice in PBS supplemented with $0.9 \mathrm{mM} \mathrm{CaCl}_{2}$ and $0.5 \mathrm{mM} \mathrm{MgCl}_{2}$ and then fixed in $2 \%$ glutaraldehyde

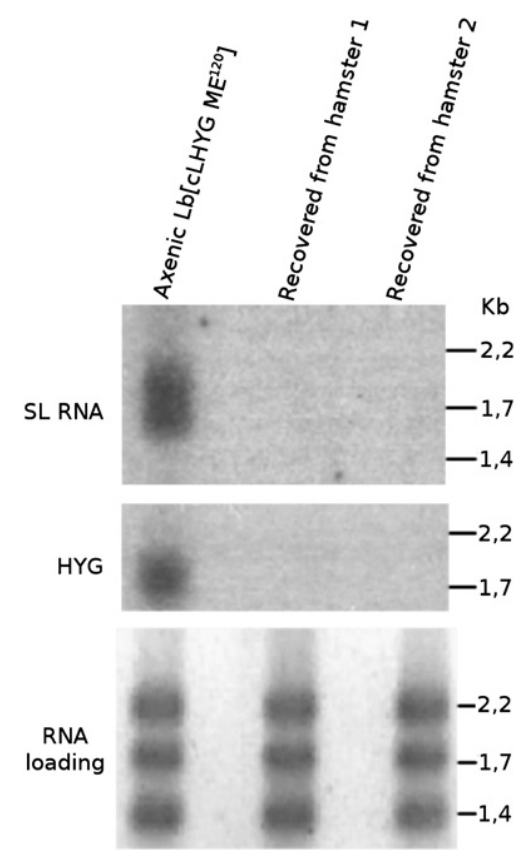

Fig. 4. Northern blotting analysis of parasites recovered from $M$. auratus footpads: lane 1) Trizol-extracted RNA from axenic transfectant Lb $\left[{ }^{c} L H Y G^{120} M E\right]$; lanes 2 and 3) Trizolextracted RNA from promastigotes recently differentiated from parasites recovered from two different hamsters after 1 year of infection. Hybridization probes: HYG, a PCR fragment of $1 \mathrm{~kb}$ containing the entire hygromycin phosphotransferase coding region; ME, a fragment of 455 bp derived from the 32D05 cosmid digested with Not I which corresponds to a entire copy of miniexon gene. Ribosomal RNA was used as a sample loading control. 
(Ladd Research Industries, Burlington, VT), 2\% paraformaldehyde (Ladd) in $0.1 \mathrm{M}$ cacodylate buffer, $\mathrm{pH} 7.4$; containing $0.05 \% \mathrm{CaCl}_{2}$, for $2 \mathrm{~h}$ at room temperature. Cells were washed twice in 0.1 cacodylate buffer, pelleted and maintained in $0.1 \mathrm{M}$ cacodylate buffer, $\mathrm{pH} 7.4$; containing $5 \%$ sucrose. Cells were post-fixed in $2 \% \mathrm{OsO}_{4}$ (Electron Microscopy Sciences, Hatfield, Pennsylvania) for $1 \mathrm{~h}$ at room temperature, rinsed in distilled water, dehydrated through a graded series of ethanol, rinsed in acetone, and embedded in EMbed 812 (Electron Microscopy Sciences, Hatfield, Pennsylvania). Sections were examined in a Phillips 208 Electron Microscope. All images were prepared for publication using The Gimp (Free Software Foundation, Boston, Massachusetts).

\section{Results}

3.1. L. (V.) braziliensis transfectants: growth behavior of axenic promastigotes

Axenic cultures of promastigotes were grown to evaluate the proliferative pattern of miniexon overexpressor as compared to control lines. All the transfectants, control lines and miniexon overexpressor exhibited growth rates and final cell densities similar to the parental L. (V.) braziliensis line (H3227) (Fig. 1). Growth behavior of these clones did not change after ten passages in axenic culture or after recovery from infected animals in the tenth week after infection (data not shown).

\subsection{Molecular characterization of $L$. (V.) braziliensis transfectants}

To investigate the correlation between miniexon transcript level and virulence pattern we transfected $L$. (V.) braziliensis (H3227) with [CLHYG ME]. Clones resistant to hygromycin B were isolated and the presence of the circular extrachromosomal molecule was confirmed by PFGE (Pulse Field Gel Electrophoresis), followed by Southern hybridization using a miniexon gene fragment as probe (data not shown). We assessed the exogenous miniexon transcript level by Northern blotting analysis. RNA from Lb [CLHYG ME] and Lb [CLHYG] transfectants was extracted in exponential and stationary growth phases, fractionated in $12 \%$ polyacrylamide gel and probed with the miniexon fragment. The results show that Lb [CLHYG ME] transfectants contain between 30 and 40\% more miniexon transcript (90 nucleotides long) than the control line, Lb [CLHYG] in both growth phases examined (Fig. 2A). In addition, the Lb [CLHYG ME] line presented a strong signal that appears as a clump in the region corresponding to 1.5-2 kb RNA molecules, a size range in which most of the Leishmania mRNA resides (Fig. 2B, lanes 4 and 5). In the same region of the blot no signal was detectable in any of the control lines, the parental line, $\mathrm{Lb}$ $\left[{ }^{2} L H Y G^{12}\right]$ and $\mathrm{Lb}\left[\mathrm{CLHYG}^{120}\right]$ under the same conditions (Fig. 2B, lanes 1,2 and 3). The Northern hybridization with the hyg probe demonstrates that the transfectants selected harbor the cosmid and that the transcription of the foreign molecule is proportional to drug pressure. The normalized densitometry values at the bottom of each panel reveal that the transgene hyg and the miniexon gene follow the same relative expression pattern. This suggests that the increase in the
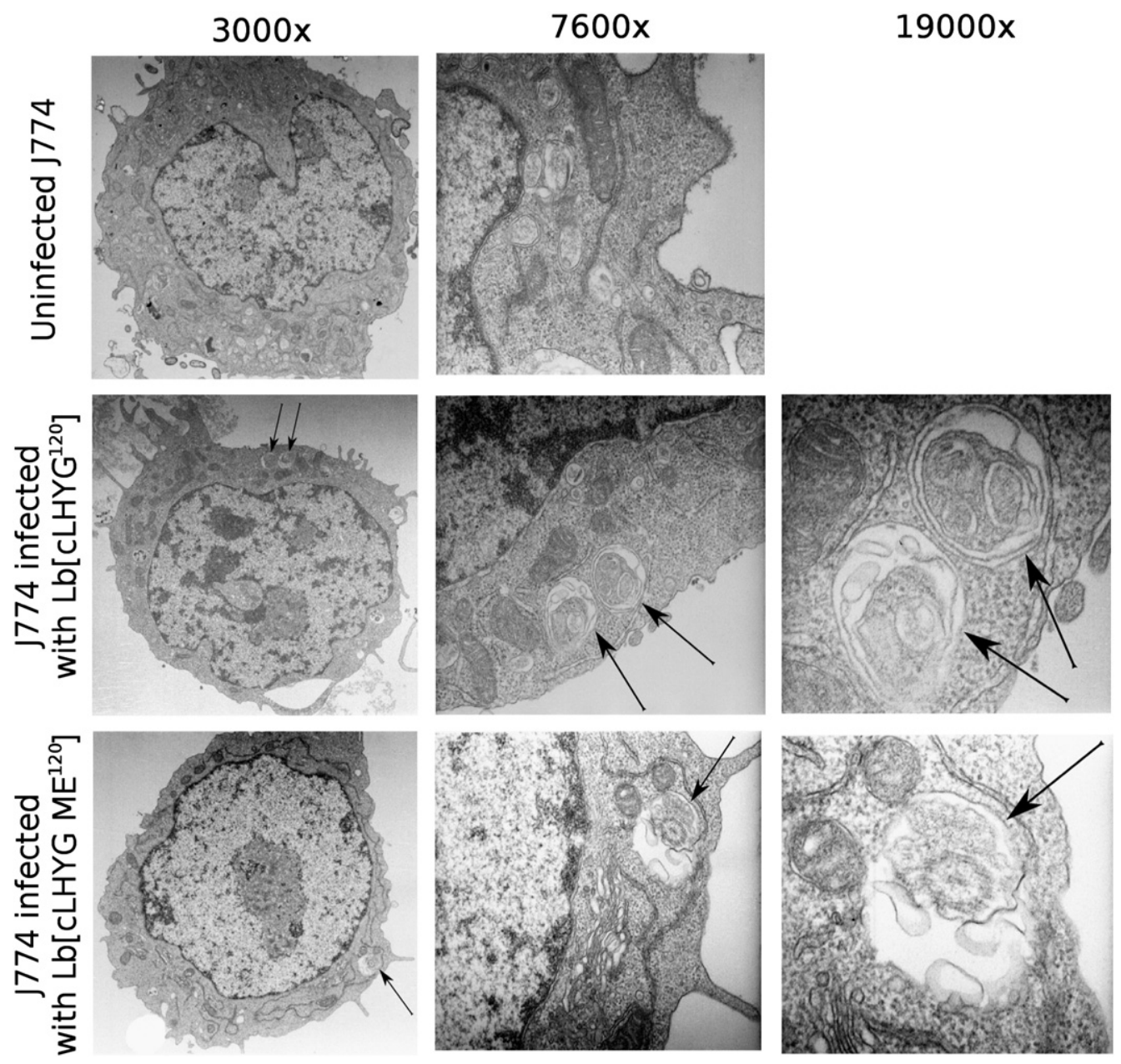

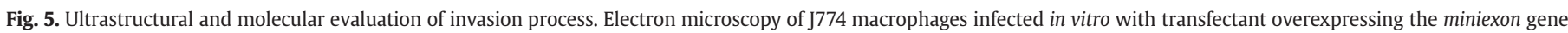
and the transfectant control. The arrows indicate the amastigotes inside parasitophorous vacuoles (3000×, 7600× and 19,000×). 
population of trans-spliced transcripts detectable in the Lb [cLHYG ME] is caused by the exogenous miniexon molecule. Northern blotting analyses indicate that the ME overexpressor presents an enhanced miniexon transcript level and that total trans-spliced mRNA levels are increased.

\subsection{Course of experimental leishmaniasis reveals that transfectants overexpressing ME RNA lose virulence in vivo}

The transfectants were cultured in 12 and $120 \mu \mathrm{g} / \mathrm{ml}$ hygromycin B in order to observe the effect of miniexon transcript surplus on the virulence. Stationary-phase promastigotes $\left(1 \times 10^{7}\right)$ were injected subcutaneously in the hind footpad of ten hamsters. The $L .(V$.$) braziliensis$ parental line (H3227) and the control transfectant, Lb [cLHYG], were used as negative control lines. Lesion development was monitored for 1 year. A dissimilar increase in footpad swelling was observed when comparing the groups for 40 weeks after infection. Hamsters inoculated with the parental line (H3227) showed progressive growth of cutaneous lesions up to 15 weeks. Lb [CLHYG] induced the development of even more severe footpad lesions than the control Lb. Therefore, neither [ $c L H Y G]$ nor hygromycin B is involved in the loss of virulence. On the other hand, hamsters inoculated with the Lb [CLHYG ME] transfectant exhibited only minor footpad swelling and failed to develop progressive infection (Fig. 3A). This phenomenon was observed after inoculation with Lb [CLHYG ME] cultured at either 12 or $120 \mu \mathrm{g} / \mathrm{ml}$ hygromycin B before infection in animal models (Fig. 3A).

We also used a novel experimental model of infection to study the ability of $L$. (V.) braziliensis parental (H3227) and transfectant lines (Lb $\left[{ }^{2} L H Y G^{120} \mathrm{ME}\right]$ and $\left.\mathrm{Lb}\left[{ }^{2} L H Y G^{120}\right]\right)$ to induce lesion development in $\mathrm{BALB} / \mathrm{c}$ mice. Animals were infected in ear dermis with $10^{5}$ parasites; the wild type $\mathrm{H} 3227$ showed a peak of parasite load 6 weeks after infection, both at the inoculation site (Fig. 3B) and in draining lymph nodes (Fig. 3C). A similar pattern was observed in animals infected with $\mathrm{Lb}\left[\mathrm{cLHYG}{ }^{120}\right]$. However, no parasites were detected in mice infected with $\mathrm{Lb}\left[\mathrm{CLHYG} \mathrm{G}^{120} \mathrm{ME}\right]$ throughout the entire period of the experiment (Fig. 3B and C), which supports the correlation between miniexon transcript excess and virulence loss. These data confirm previous findings [15] and extend the positive correlation between extrachromosomal overexpression of the miniexon gene and virulence attenuation to $L$. $(V$.) braziliensis.

3.4. Molecular analysis of parasites recovered from animals confirms the correlation between miniexon transcript excess and attenuation

Infected hamsters were monitored for 1 year. After this period two out of ten animals from the Lb [CLHYG ME] group presented a small lesion on the infected footpad. This finding led us to investigate a possible ME transcript level decrease in parasites recovered from these animals. Amastigotes were rescued from the footpads and total RNA was extracted from recently differentiated promastigotes. The RNA was fractionated in denaturing agarose gels and transferred to nylon membranes for Northern blot analysis. We observed that parasites recovered from these lesions no longer overexpressed the hygromycin phosphotransferase or the exogenous miniexon gene (Fig. 4). This result confirms that the excess of ME transcripts negatively affects the virulence of Leishmania during the infection of a mammalian host. In addition, we demonstrated that the miniexon overexpressor transfectant maintains its ability to infect and differentiate within J774 macrophages in vitro (Fig. 5).

\section{Discussion}

In this study we report the influence of miniexon expression levels on virulence patterns of $L$. $(V$.) braziliensis. The availability of a cosmid bearing 100 repeats of the $L$. (L.) major miniexon gene and wellestablished infection protocols, together with our ability to stably introduce cosmids into $L$. $(V$.) braziliensis, made it possible to correlate the artificial overexpression of ME with virulence decrease. We worked with cloned transfectants, selected as colonies from solid medium supplemented with hygromycin B. Two animal models with different genetic and immune backgrounds were infected with these clones, and two modes of infection were used (subcutaneous and intradermal injection). Working with large groups of animals and independent experiments, we observed the attenuation of $L .(V$. braziliensis ME overexpressor in all cases.

This investigation extends a previous study by our group with similar results in $L$. (L.) major [15]. Therefore, the observed phenomenon occurs within two distinct subgenera. Virulence attenuation is more noticeable in $L .(V$.) braziliensis transfectants, as no lesion is observed even using one tenth of the drug concentration necessary to attenuate $L$. ( $L$.) major [cLHYG ME]. Previous and present data allow us to associate miniexon overexpression with loss of virulence.

Conflicting data have been reported by Zhang and Matlashewski [22]. They have recently described an increased virulence of $L$. (L.) major transfectants harboring either a $L$. $(L$.) donovani miniexon gene array or the [CLHYG ME] construct. A drawback of their study is that a population of resistant cells was used instead of clonal lines. Although these authors recovered virulent transfectants from infected animals, they did not report whether the loss of the extrachromosomal molecule leads to the reversion of the phenotype, a crucial experimental step in order to establish the correlation between the transfected molecule and virulence. Therefore, the experimental design adopted by Zhang and Matlashewski [22] does not allow exclusion of a virulence variation unrelated to the presence of ME cosmid. In addition, it is known that variability of parasite fitness can occur naturally due to unknown factors, leading to an apparently spontaneous virulence reversion. This phenomenon has been described by different authors and represents an obstacle to the use of naturally avirulent lines as live vaccines $[15,23]$. The dynamics of virulence in these parasites is complex and this poorly understood issue could be the result of a combination of factors, including epigenetic ones [24].

Our previous data from the $L$. (L.) major ME overexpressor showed that the "cure" of the extrachromosomal molecule leads to a reversion to the parental phenotype. This study with $L$. $(V$.) braziliensis has added relevant data to better understand the attenuation induced by overexpression of the miniexon. The persistence of the parasite and the long-lasting effect of ME overexpression are important features revealed here. We have demonstrated that the $L$. $(V$.) braziliensis ME transfectant, rescued from hamsters after 1 year of infection showed undetectable levels of expression of the exogenous genes. Nevertheless, these parasites could grow in axenic culture supplemented with hygromycin B, which suggests that the cosmid is present in at least some of the cells of the population. The subtle clinical manifestation induced by this attenuated parasite after more than 40 weeks of hamster infection shows that $\mathrm{Lb}\left[\mathrm{CLHYG}^{120} \mathrm{ME}\right]$ does not mimic the virulence pattern of the parental line. Therefore, the parasite persistence in infected hamsters and the successful infection of J774 macrophages supports the hypothesis that the inability of the mutant to cause disease is due to an impairment of amastigotes to proliferate rather than a failure to invade the host cell. Although an impairment of Lb [CLHYG ME] to infect BALB/c cells could be an explanation for the undetectable levels of parasites in these animals this is not supported by our current and previous data [15].

Leishmania mutants overexpressing the miniexon gene could be a suitable model for further studies on host-parasite interactions and could be useful to better understand some aspects of virulence and the parasite's needs to proliferate within macrophages.

Presented results suggest that the miniexon mutant has an increased activity of trans-splicing which leads to high levels of trans-spliced transcripts. Presented data associated with the fact that high levels of drug pressure lead to an increased expression of all the 
genes of recombinant cosmids [26] indicate that the miniexon gene is overexpressed in this mutant. We may postulate that the probable overexpression of the exogenous miniexon may lead to an imbalance of translated product levels, which should be down-regulated in amastigotes and crucial for the proliferation of this intracellular form. The imbalance generated is incompatible with the parasite's intracellular life in the hostile phagolysosome. Further studies should attempt to answer these questions.

We have demonstrated that $L .(V$.) braziliensis transfectants overexpressing the spliced leader RNA lose their original virulent pattern, similarly to what had been previously observed in $L$. $(L$.) major ME overexpressors. Besides extending previous findings to another subgenus, we have shown here that these attenuated parasites are persistent within the host. These mutants may be important for the generation of safe vaccines and are also useful tools for the study of the control of gene expression routes in Leishmania.

\section{Acknowledgments}

We thank Viviane A. Trombela and Fernanda O. Novais for technical assistance. This work was supported by Fundação de Amparo à Pesquisa do Estado de São Paulo (FAPESP-99/12403-3) and Conselho Nacional de Desenvolvimento Científico e Tecnológico. AFJS and JST were supported by fellowships from FAPESP (01/092815 and 03/02366-0).

\section{References}

[1] Desjeux P. Leishmaniasis. Nat Rev Microbiol 2004;2(9):692.

[2] Beverley SM. Protozomics: trypanosomatid parasite genetics comes of age. Nat Rev Genet 2003;4(1):11-9.

[3] Clayton CE. Life without transcriptional control? From fly to man and back again. Embo J 2002;21(8):1881-8.

[4] Campbell DA, Thornton DA, Boothroyd JC. Apparent discontinuous transcription of Trypanosoma brucei variant surface antigen genes. Nature 1984;311(5984):350-5.

[5] Cook GA, Donelson JE. Mini-exon gene repeats of Trypanosoma (Nannomonas) congolense have internal repeats of 190 base pairs. Mol Biochem Parasitol 1987;25(1): 113-22.

[6] Fernandes O, Murthy VK, Kurath U, Degrave WM, Campbell DA. Mini-exon gene variation in human pathogenic Leishmania species. Mol Biochem Parasitol 1994;66(2): 261-71.

[7] Spithill TW, Samaras N. Genomic organization, chromosomal location and transcription of dispersed and repeated tubulin genes in Leishmania major. Mol Biochem Parasitol 1987;24(1):23-37.
[8] Wincker P, Ravel C, Blaineau C, Pages M, Jauffret Y, Dedet JP, et al. The Leishmanid genome comprises 36 chromosomes conserved across widely divergent human pathogenic species. Nucleic Acids Res 1996;24(9):1688-94.

[9] Ivens AC, Peacock CS, Worthey EA, Murphy L, Aggarwal G, Berriman M, et al. The genome of the kinetoplastid parasite, Leishmania major. Science 2005;309(5733): 436-42.

[10] LeBowitz JH, Smith HQ, Rusche L, Beverley SM. Coupling of poly(A) site selection and trans-splicing in Leishmania. Genes Dev 1993;7(6):996-1007.

[11] Samaras N, Spithill TW. Molecular karyotype of five species of Leishmania and analysis of gene locations and chromosomal rearrangements. Mol Biochem Parasitol 1987;25(3):279-91.

[12] Ullu E, Matthews KR, Tschudi C. Temporal order of RNA-processing reactions in trypanosomes: rapid trans splicing precedes polyadenylation of newly synthesized tubulin transcripts. Mol Cell Biol 1993;13(1):720-5.

[13] Iovannisci DM, Beverley SM. Structural alterations of chromosome 2 in Leishmania major as evidence for diploidy, including spontaneous amplification of the miniexon array. Mol Biochem Parasitol 1989;34(2):177-88.

[14] Bastien P, Blaineau C, Britto C, Dedet JP, Dubessay P, Pages M, et al. The complete chromosomal organization of the reference strain of the Leishmania Genome Project, L. major 'Friedlin'. Parasitol Today 1998;14(8):301-3.

[15] Antoniazi S, Lima HC, Cruz AK. Overexpression of miniexon gene decreases virulence of Leishmania major in BALB/c mice in vivo. Mol Biochem Parasitol 2000;107(1):57-69.

[16] Kapler GM, Coburn CM, Beverley SM. Stable transfection of the human parasite Leishmania major delineates a 30-kilobase region sufficient for extrachromosomal replication and expression. Mol Cell Biol 1990;10(3):1084-94.

[17] Clayton C, Adams M, Almeida R, Baltz T, Barrett M, Bastien P, et al. Genetic nomenclature for Trypanosoma and Leishmania. Mol Biochem Parasitol 1998;97(1-2): 221-4.

[18] Sambrook J, Fritsch EF, Maniatis T. Molecular Cloning: A Laboratory Manual. NY: Cold Spring Harbor: Cold Spring Harbor Laboratory Press, 1989.

[19] Feinberg AP, Vogelstein B. A technique for radiolabeling DNA restriction endonuclease fragments to high specific activity. Anal Biochem 1983;132(1):6-13.

[20] Feinberg AP, Vogelstein B. "A technique for radiolabeling DNA restriction endonuclease fragments to high specific activity". Addendum. Anal Biochem 1984;137(1):266-7.

[21] Titus RG, Marchand M, Boon T, Louis JA. A limiting dilution assay for quantifying Leishmania major in tissues of infected mice. Parasite Immunol 1985;7(5):545-55.

[22] Zhang WW, Matlashewski G. In vivo selection for Leishmania donovani miniexon genes that increase virulence in Leishmania major. Mol Microbiol 2004;54(4): 1051-62.

[23] Green MS, Kark JD, Witztum E, Greenblatt CL, Spira DT. Frozen stored Leishmania tropica vaccine: the effects of dose, route of administration and storage on the evolution of the clinical lesion. Two field trials in the Israel Defense Forces. Trans $\mathrm{R}$ Soc Trop Med Hyg 1983;77(2):152-9.

[24] Ebert D. Experimental evolution of parasites. Science 1998;282(5393):1432-5.

[25] de Moura TR, Novais FO, Oliveira F, Clarencio J, Noronha A, Barral A, et al. Toward a novel experimental model of infection to study American cutaneous leishmaniasis caused by Leishmania braziliensis. Infect Immun 2005;73(9):5827-34.

[26] Pedrosa AL Cruz AK. The effect of location and direction of an episomal gene on the restoration of a phenotype by functional complementation in Leishmania. Mol Biochem Parasitol 2002;122(2):141-8. 